\title{
A Practical Iron-based Newman-Kwart Rearrangement Under Oxidative Conditions
}

\author{
Thibault Gendron, ${ }^{\mathrm{a}, \mathrm{b}, \mathrm{t}}$ Raul Pereira, ${ }^{\mathrm{c}, \mathrm{d}, \mathrm{t}}$ Hafsa Y. Abdi, Timothy H. Witney, ${ }^{\mathrm{c}, \mathrm{d},{ }^{*}}$ and Erik Årstad ${ }^{\mathrm{a}, \mathrm{b},{ }^{*}}$

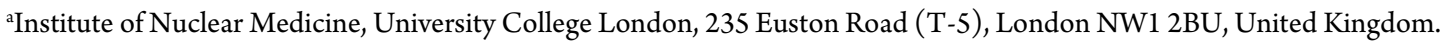 \\ bepartment of Chemistry, University College London, 20 Gordon Street, London WC1H 0AJ, United Kingdom. \\ ${ }^{\mathrm{c}}$ Centre for Advanced Biomedical Imaging, University College London, 72 Huntley Street, London WC1E 6DD, United Kingdom. \\ 'Imaging Chemistry and Biology, King's College London, St. Thomas' Hospital, London SE1 7EH, United Kingdom. \\ + TG and RP contributed equally to the work. * TW and EA jointly supervised this work. \\ Corresponding authors: tim.witney@kcl.ac.uk and e.arstad@ucl.ac.uk
}

\begin{abstract}
Herein, we report that iron(II)/ammonium persulfate in aqueous acetonitrile mediates the NewmanKwart rearrangement of $O$-aryl carbamothioates. Electron-rich substrates react rapidly under moderate heating to afford the rearranged products in excellent yields. The mild conditions, rapid reaction rates, and suitability for scale up offers immediate practical benefits to access functionalised thiophenols.
\end{abstract}

The Newman-Kwart rearrangement (NKR) - the transformation of $O$-aryl carbamothioates to the corresponding $S$-aryl carbamothioates - gives access to thiophenols from their more readily available phenol counterparts. ${ }^{1,2}$ The three-step sequence, which involves phenol protection with thiocarbamoyl chloride, NKR and deprotection of the resulting carbamothioate, is appealing as it avoids the need for highly reactive reagents or handling of foul-smelling chemicals. The NKR is therefore a synthetically important reaction with widespread

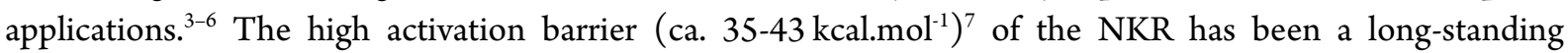
limitation as thermal activation requires temperatures of $150^{\circ} \mathrm{C}$ for electron-deficient substrates to $>300^{\circ} \mathrm{C}$ for non-activated arenes (Figure 1).?

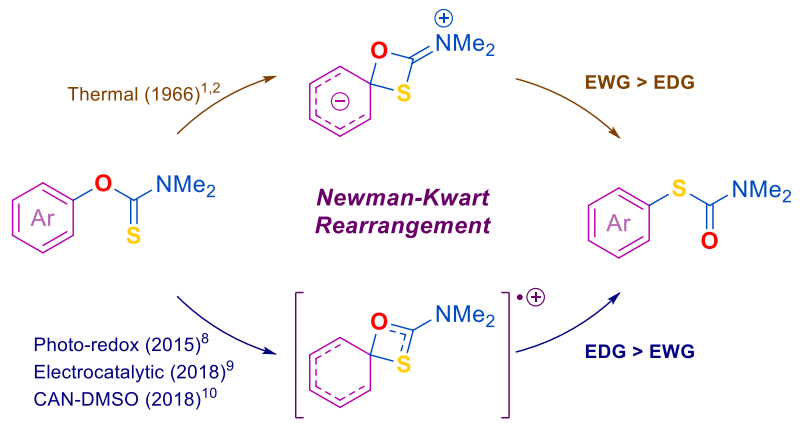

Figure 1. The Newman-Kwart Rearrangement (NKR). Abbreviation: EWG: electron-withdrawing groups; EDG: electron-donating groups.

At such high temperatures, compound volatility, decomposition and charring becomes problematic. In practice, the thermal reaction is therefore limited to activated, thermally stable and non-volatile substrates. Renewed interest in the NKR has led to the discovery of several catalytic systems that favours electron rich substrates, including a photo-redox catalytic system, ${ }^{8}$ an electrochemical method, ${ }^{9}$ and more recently, a chemical reaction involving single-electron oxidation of $S$-aryl carbamothioates with ceric-ammonium nitrate (CAN) in dimethylsulfoxide (DMSO). ${ }^{10}$ The latter method makes the NKR with electron-rich substrates widely accessible as it overcomes the need for specialist equipment. However, the use of DMSO as the solvent, and the need for high substrate dilution, practically limits applications to small-scale reactions.

As a part of our ongoing research program, we needed a robust scalable method to access $S$ - $\left(3^{\prime}, 5^{\prime}-\right.$ dimethoxy-5-methyl-[1,1'-biphenyl]-2-yl) dimethylcarbamothioate $\mathbf{2 a}$ from the corresponding $O$-aryl carbamothioate 1a (Scheme 1). ${ }^{11}$ Although successful on small scale, the thermal NKR proved operationally challenging to scale to multi-gram quantities as inconsistent heating resulted in variable yields. 

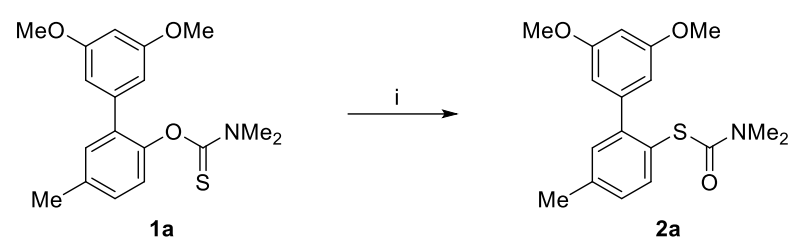

Scheme 1. Preliminary results. Conditions: silver nitrate ( $35 \mathrm{~mol} \%)$, ammonium persulfate (1.3 equiv.), acetonitrile/water $3: 1,85^{\circ} \mathrm{C}, 90 \mathrm{~min}, 78 \%$ yield.

Attempted Pd-catalysed NKR only afforded trace amounts of product $\mathbf{2 a}$ in agreement with the previously reported scope. ${ }^{12}$ Inspired by the work of Anderson and Kochi on radical decarboxylation of carboxylic acids, ${ }^{13}$ we attempted to use silver nitrate and ammonium persulfate (APS) as a single electron oxidant to mediate this transformation. Gratifyingly, under these conditions ( $35 \mathrm{~mol} \% \mathrm{AgNO}_{3}, 1$ equiv. APS, $\mathrm{CH}_{3} \mathrm{CN} / \mathrm{H}_{2} \mathrm{O}, 85^{\circ} \mathrm{C}$ ) $\mathbf{1 a}$ rearranged to target product $\mathbf{2 a}$ in $78 \%$ yield (Scheme 1). In a bid to develop a practical and scalable method, we investigated the effect of the different reagents and reaction parameters using O-(4-methoxyphenyl) dimethylcarbamothioate $\mathbf{1 b}$ as a model compound (Table 1).

Table 1: Optimisation of the novel NKR protocol

\begin{tabular}{ccccccc} 
No & $\begin{array}{c}\text { Equiv. } \\
\text { of APS }\end{array}$ & $\begin{array}{c}\text { Metal } \\
(\mathrm{mol} \%)\end{array}$ & Solvents & $\begin{array}{c}\mathrm{Temp} \\
\left({ }^{\circ} \mathrm{C}\right)\end{array}$ & $\begin{array}{c}\text { Conc. } \\
(\mathrm{M})\end{array}$ & $\begin{array}{c}\text { Conv. } \\
(\%)^{a}\end{array}$ \\
\hline 1 & 1.3 & $\mathrm{Ag}(35)^{b}$ & $\mathrm{CH}_{3} \mathrm{CN} / \mathrm{H}_{2} \mathrm{O} 3: 1$ & 85 & 0.083 & $>95$ \\
2 & 0 & $\mathrm{Ag}(35)^{b}$ & $\mathrm{CH}_{3} \mathrm{CN} / \mathrm{H}_{2} \mathrm{O} 3: 1$ & 85 & 0.083 & $<5$ \\
3 & 1 & - & $\mathrm{CH}_{3} \mathrm{CN} / \mathrm{H}_{2} \mathrm{O} 3: 1$ & 85 & 0.083 & $10-95^{d}$ \\
4 & 1 & $\mathrm{Fe}(5)^{c}$ & $\mathrm{CH}_{3} \mathrm{CN} / \mathrm{H}_{2} \mathrm{O} 3: 1$ & 85 & 0.083 & $>95$ \\
5 & 1 & $\mathrm{Fe}(5)^{c}$ & $\mathrm{CH}_{3} \mathrm{CN} / \mathrm{H}_{2} \mathrm{O} 3: 1$ & 45 & 0.083 & $>95(91)$ \\
6 & 1 & $\mathrm{Fe}(5)^{c}$ & $\mathrm{CH}_{3} \mathrm{CN} / \mathrm{H}_{2} \mathrm{O} 3: 1$ & 45 & 0.25 & 10 \\
7 & 1 & $\mathrm{Fe}(5)^{c}$ & $\mathrm{CH}_{3} \mathrm{CN} / \mathrm{H}_{2} \mathrm{O} 3: 1$ & 45 & 0.17 & $>95$ \\
8 & 1 & $\mathrm{Fe}(5)^{c}$ & $\mathrm{CH}_{3} \mathrm{CN}$ & 65 & 0.083 & $>95^{d}$ \\
& & & & & & \\
\hline
\end{tabular}

${ }^{a}$ As determined by ${ }^{1} \mathrm{H}$ NMR, isolated yields are given in brackets; ${ }^{b}$ As silver nitrate; ${ }^{c}$ As Mohr's salt; ${ }^{d}$ Depending on the source of APS; ${ }^{e}$ Reaction was heated for four hours, conversion to $\mathbf{3 b}$.

When subjected to the aforementioned conditions, $\mathbf{1 b}$ was fully converted to $\mathbf{2} \mathbf{b}$ (Table 1 , entry 1 ). Control experiments proved that APS is essential for the reaction to proceed (Table 1, entry 2). In the absence of silver, we observed large variations in yields depending on the source of APS. Subsequent analysis by inductively coupled plasma mass spectrometry (ICP-MS) revealed high levels of iron in the batch of APS that most effectively mediated transformation of $\mathbf{1 b}$ to $\mathbf{2} \mathbf{b}$. As iron is known to accelerate the decomposition of APS in a similar manner to silver, ${ }^{13,14}$ we hypothesized that the iron impurity played a key role in the reaction. Gratifyingly, replacing silver nitrate with catalytic amounts $(5 \mathrm{~mol} \%)$ of Mohr's salt $\left(\left(\mathrm{NH}_{4}\right)_{2} \mathrm{Fe}\left(\mathrm{SO}_{4}\right)_{2} \cdot 6 \mathrm{H}_{2} \mathrm{O}\right)$ afforded $\mathbf{2 b}$ in $95 \%$ yield (Table 1, entry 4). Lowering the reaction temperature from $85^{\circ} \mathrm{C}$ to $45^{\circ} \mathrm{C}$ still gave full conversion within an hour and afforded $\mathbf{2 b}$ in $91 \%$ isolated yield (Table 1, entry 5). As described in the previously reported non-thermal NKR protocols, ${ }^{8-10}$ the reaction proved less efficient at high concentrations. At a concentration up to $0.17 \mathrm{M}$ (Table 1, entry 7), the rate of transformation appeared to be unaffected, however, at $0.25 \mathrm{M}$ the yield dropped to $10 \%$ under otherwise identical conditions (Table 1, entry 6). Finally, the use of water as co-solvent proved crucial for the formation of the target product. Indeed, when acetonitrile was used as the sole reaction solvent, starting material $\mathbf{1 b}$ was converted quantitatively to the corresponding carbamate $\mathbf{3} \mathbf{b}$ (Table 1 , entry 8). With optimised conditions in hand (5 mol\% Mohr's salt, 1 equiv. APS, $\mathrm{CH}_{3} \mathrm{CN} / \mathrm{H}_{2} \mathrm{O}$ 3:1), we explored the scope of this novel NKR reaction (Figure 2). 


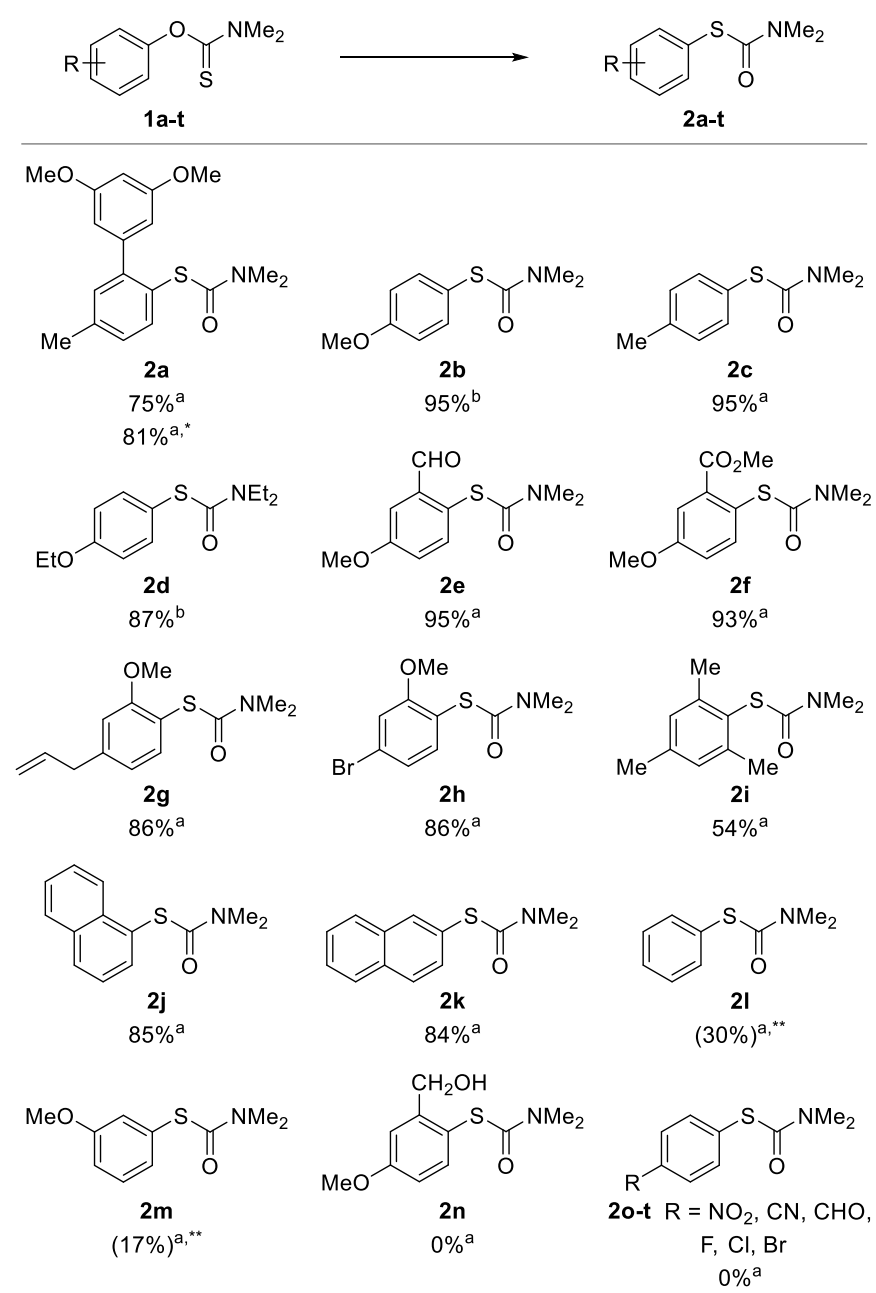

Figure 2: Scope study. Conditions: ${ }^{a} \mathbf{1}(1 \mathrm{mmol})$, Mohr's salt (5 mol\%), APS ( 1 equiv.), $\mathrm{CH}_{3} \mathrm{CN} / \mathrm{H}_{2} \mathrm{O} 3: 1,65^{\circ} \mathrm{C}, 2 \mathrm{~h}$; ${ }^{b} \mathbf{1}$ ( $1 \mathrm{mmol}$ ), Mohr's salt $\left(5 \mathrm{~mol} \%\right.$ ), APS (1 equiv.), $\mathrm{CH}_{3} \mathrm{CN} / \mathrm{H}_{2} \mathrm{O} 3: 1,45^{\circ} \mathrm{C}, 1 \mathrm{~h}$; ${ }^{*}$ Scale-up to $10 \mathrm{mmol}$; ${ }^{* *}$ Conversion determined by ${ }^{1} \mathrm{H}$ NMR.

Substrates substituted with electron donating groups (EDG) in para-position afforded rearranged products 2a-f in nearly quantitative yields. Additional electron-withdrawing groups (EWG) were well tolerated as exemplified with the formation of aldehyde and ester substituted products $\mathbf{2 e}$ and $\mathbf{2} \mathbf{f}$ in $95 \%$ and $93 \%$ yields, respectively. Steric hindrance had little-to-no influence on the rearrangement, as ortho-substituted products $\mathbf{2 a}$ and $\mathbf{2 f - i}$ were obtained in good to excellent yields. The reaction displayed a good functional group tolerance as aldehyde, ester, allyl and bromo substituents in products $\mathbf{2 e - h}$ remained intact through the procedure; notably, oxidation of aldehyde $2 \mathbf{e}$ was not observed. However, rearrangement of benzylic alcohol $\mathbf{2 n}$ was problematic due to the oxidation of the alcohol to the corresponding aldehyde, resulting in the formation of a complex mixture of products. $S$-(naphthalene-1-yl) dimethyl-carbamothioate $\mathbf{2} \mathbf{j}$ as well as its 2 -regioisomer $\mathbf{2} \mathbf{k}$ were obtained in $85 \%$ and $84 \%$ isolated yields, respectively. This result is of note as the CAN,${ }^{10}$ photoredox ${ }^{8}$ and electrocatyltic ${ }^{9}$ methods allow access to the 1-napthalene but not the 2-napthalene derivative. Formation of electron-neutral 21 and moderately electron-deficient meta-methoxy substituted $\mathbf{2 m}$ was observed, albeit in modest conversions ( $30 \%$ and $17 \%$, respectively). Attempted reactions with electron-deficient substrates proved troublesome; nitro- 1o, nitrile- 1p, aldehyde- 1q, and halide- 1r-t substituted $O$-aryl carbamothioate failed to rearrange. In most cases, NMR analysis of the reaction mixture showed that the starting materials were transformed to the corresponding $\mathrm{O}$-aryl carbamates instead of the expected $\mathrm{S}$-aryl carbamothioate. Formation of carbamates has previously been reported for the CAN-DMSO mediated NKR reaction. ${ }^{10}$ 
To get a better understanding of this side reaction, isotopically labelled $\left[{ }^{18} \mathrm{O}\right] \mathrm{O}$-aryl carbamothioate ${ }^{18} \mathrm{O}-2 \mathrm{o}$ was subjected to the reaction conditions with strict exclusion of water and oxygen (Scheme $2 \mathrm{~A}$ ). Carbamate ${ }^{18} \mathrm{O}-3 \mathbf{j}$ was isolated in $60 \%$ yield. Tandem mass spectrometry (MS/MS) confirmed the position of the $\left[{ }^{18} \mathrm{O}\right]$ oxygen on the molecule as shown on Scheme $2 \mathrm{~A}$. In the absence of any other source of oxygen, this demonstrates that the extra oxygen added on the carbamate is likely to come from the persulfate. Furthermore, subjecting $\mathbf{l b}$ to the standard reaction conditions whilst replacing $\mathrm{H}_{2} \mathrm{O}$ with $\left[{ }^{18} \mathrm{O}\right] \mathrm{H}_{2} \mathrm{O}$ did not lead to any isotopic exchange on the rearranged product, thus suggesting that water is not actively participating in the reaction (Scheme 2B).

A]<smiles>CN(C)C(=S)Oc1ccc([N+](=O)[O-])cc1</smiles>

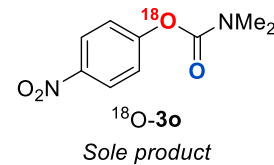

Sole product

B]

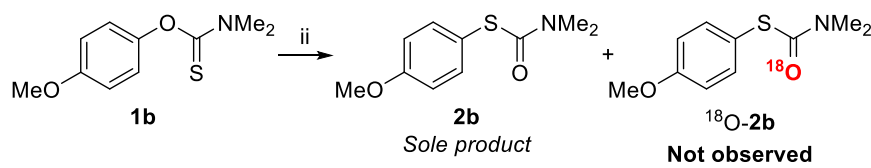

c]
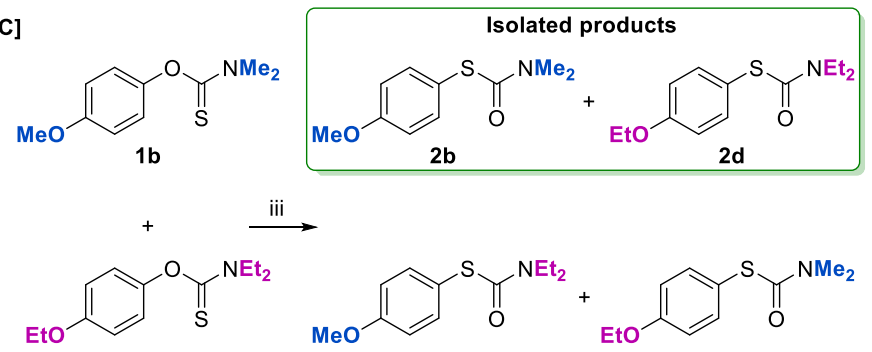

1d

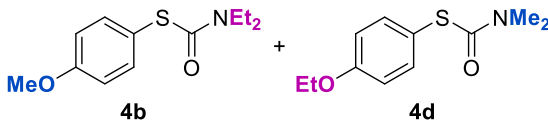

Crossover products - Not observed

Scheme 2: Isotopic labelling experiments (A, B) and crossover experiment (C). Conditions: (i) Mohr's salt (5 mol\%), APS (2 equiv.), anhydrous degassed $\mathrm{CH}_{3} \mathrm{CN}, 65^{\circ} \mathrm{C}, 3 \mathrm{~h}$; (ii) Mohr's salt (5 mol\%), APS (1 equiv.), $\mathrm{CH}_{3} \mathrm{CN} /\left[{ }^{18} \mathrm{O}\right] \mathrm{H}_{2} \mathrm{O} 3: 1,45^{\circ} \mathrm{C}, 1 \mathrm{~h},(\mathrm{iii}) \mathrm{Mohr}$ 's salt (5 mol\%), APS (1 equiv.), $\mathrm{CH}_{3} \mathrm{CN} / \mathrm{H}_{2} \mathrm{O} 3: 1,45^{\circ} \mathrm{C}, 1 \mathrm{~h}$.

Overall, the results of this scope study are in line with the works previously published on oxidative NKR: the reaction proceeded rapidly with electron rich ring systems, non-activated systems reacted more sluggishly, while electron-deficient substrates failed to react, or underwent a side-reaction to give the corresponding $O$ carbamates.

To elucidate the rearrangement mechanism itself, we first focused our attention on the reaction kinetics. ${ }^{1} \mathrm{H}$ NMR reaction monitoring of the para-methoxy derivative $\mathbf{1 b}$ led to a sigmoidal kinetic profile. After an induction period of about $35 \mathrm{~min}, \mathbf{1} \mathbf{b}$ was quantitatively rearranged to product $\mathbf{2} \mathbf{b}$ within $20 \mathrm{~min}$ on a $0.5 \mathrm{mmol}$ scale (zero order linear approximation $k \approx 5 \mathrm{mmol} \cdot \mathrm{L}^{-1} \cdot \mathrm{min}^{-1}$ ). Although not uncommon, sigmoidal kinetic profiles are difficult to interpret; unrevealing which mechanisms are responsible for the induction period and then for reaction lift-off is challenging and outside of the scope of the present study. We subsequently investigated whether the reaction was inter- or intramolecular through a crossover experiment between the para-methoxy derivative $\mathbf{1 b}$ and its ethyl analogue $\mathbf{1 d}$ (Scheme 2C). Should the reaction be intermolecular, an interchange of substituents would occur, giving rise to crossover rearranged products $\mathbf{4 b}$ and $\mathbf{4 d}$. NMR analysis of the crude reaction mixture showed exclusive formation of the two non-crossover rearranged products $\mathbf{2 b}$ and 2d, in equal amounts. This observation suggests the reaction to be solely intramolecular, matching the results previously obtained with the CAN-mediated NKR method. ${ }^{10}$

The observed reactivity (Figure 2) is consistent with the formation of a radical-cation transition state as previously reported. ${ }^{8-10}$ In aqueous conditions, iron(II)/(III) and iron oxides are known to mediate Fentonlike process, resulting in single electron transfer. Under such conditions, APS decomposes to the sulfate radical anion $\mathrm{SO}_{4}{ }^{-} \cdot{ }^{15}$ Adding in the reaction an equivalent of (2,2,6,6-tetramethylpiperidin-1-yl)oxyl (TEMPO), an established radical trap, blocked the rearrangement, supporting the hypothesis of a radical-mediated reaction. In addition, close inspection of the crude rearrangement mixture consistently revealed the presence of a dark orange-brown residue consistent with the formation of iron oxides. On the basis of these observations, we propose the mechanism illustrated in Scheme 3. A Fenton-like process results in the formation of sulfate radical anion $\mathrm{SO}_{4}^{-\bullet}$ (Scheme 3, blue box), which, in turn, reacts by abstracting an electron from the sulfur atom in $\mathbf{1 b}$, forming radical cation $\mathbf{1 b}^{+\bullet}$. Subsequent intramolecular (vide supra) rearrangement of $\mathbf{1 b}^{+\bullet}$ leads to the formation of a putative four-center intermediate $\mathbf{I}$, as previously described. ${ }^{16}$ 


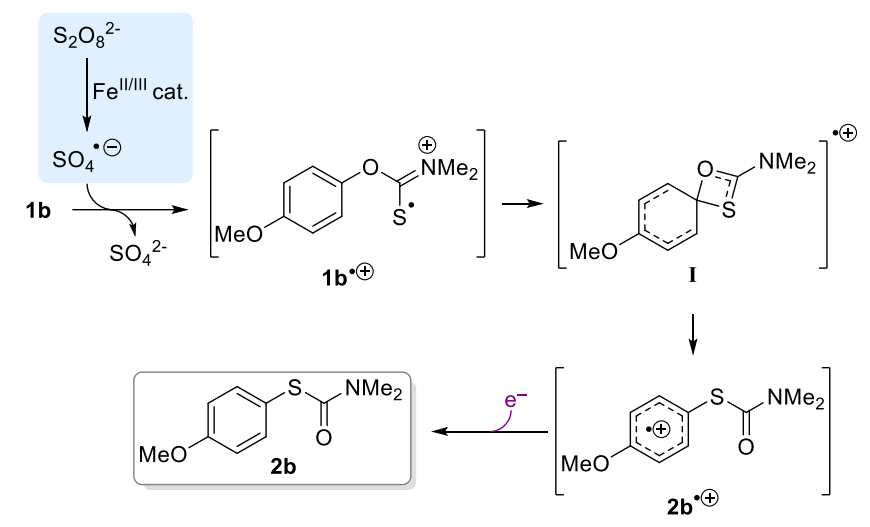

Scheme 3: Proposed mechanism.

Heterolytic cleavage of the $O_{\text {Aryl }}$ bond results in radical cation $\mathbf{2 b}^{+\bullet}$. The rearranged product $\mathbf{2 b}$ is then obtained by single electron reduction of radical cation $\mathbf{2 b}^{+\boldsymbol{\bullet}}$. The exact nature of the reduction step is unclear; it is possible that $\mathbf{l b}$ serves as an electron donor, thereby regenerating $\mathbf{1 b}^{+\bullet}$. However, the experimental observations are not consistent with a radical chain reaction. It is therefore more likely that the single electron reduction is mediated by the iron(II)/(III)-persulfate system.

To demonstrate a practical application, the novel strategy was used in the synthesis of the labelling precursor of ${ }^{18} \mathrm{~F}-\mathrm{AEM} 1,{ }^{17}$ a radiotracer of interest for the imaging of cancer drug resistance by positron emission tomography (Scheme 4). The synthesis of $\mathbf{2 a}$ was successfully scaled-up to $3 \mathrm{~g}(10 \mathrm{mmol})$, affording the target rearranged product in $81 \%$ yield. Biaryl building block 2 a was then coupled to 5 giving biaryl thioether 6 in $60 \%$ yield. Subsequent ring-closing sulfonium salt formation ${ }^{11}$ afforded labelling precursor 7 in $72 \%$ yield. Subsequent labelling of 7 with fluorine-18 (DMSO, $125^{\circ} \mathrm{C}, 25 \mathrm{~min}$, non-optimised) afforded ${ }^{18} \mathrm{~F}$-AEM1 in $15 \pm 4 \%(n=4)$ decay-corrected radiochemical yield (d.c. RCY).
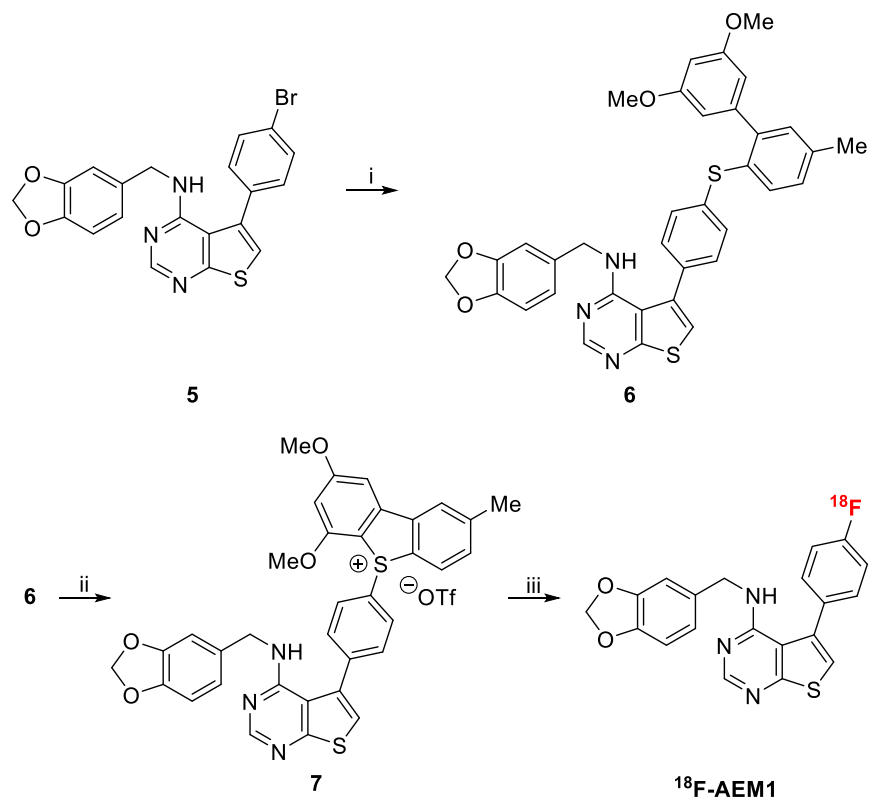

Scheme 4: Application the labelling of ${ }^{18} \mathrm{~F}$-AEM1. Conditions: (i) $\mathbf{2 a}, t \mathrm{BuOK}, \mathrm{Pd}_{2}\left(\mathrm{dba}_{3}\right.$, DPEPhos, toluene, reflux, $56 \%$ yield; (ii) $\mathrm{Ca}(\mathrm{OCl})_{2}$, acetate buffer $\mathrm{pH} 4$, acetonitrile, $3^{\circ} \mathrm{C}, 15 \mathrm{~min}, 72 \%$ yield; (iii) ${ }^{18} \mathrm{~F}^{-}, \mathrm{K}_{222} / \mathrm{KHCO}_{3}, \mathrm{DMSO}, 125^{\circ} \mathrm{C}$, $25 \min , 15 \pm 4 \%$ d.c. $\operatorname{RCY}(n=4)$.

In conclusion, we report that catalytic amounts of $\mathrm{Fe}(\mathrm{II})$ in the presence of APS mediates conversion of electron-rich and electron neutral $O$-aryl carbamothioates to the corresponding $S$-aryl carbamothioates under mild conditions. The reaction has a similar scope to previously reported methods for cation-radical NKR, but offers clear practical advantages in that it circumvents the need for specialist equipment, proceeds with shorter reaction times and at higher substrate concentration, and the use of a volatile solvent makes it well suited for larger-scale reactions. 


\section{Acknowledgements}

The research leading to these results was funded by the European Union's Seventh Framework Programme (FP7/2007-2013) under grant agreement $n^{\circ} 602102$ (EPITARGET) (EA), a Wellcome Trust and Royal Society Sir Henry Dale Fellowship (107610/Z/15/Z) to (THW), CRUK \& EPSRC Comprehensive Cancer Imaging Centre at KCL, UCL \& Imperial jointly funded by Cancer Research UK and the Engineering and Physical Sciences Research Council (EPSRC; C1519/A16463; C2536/A10337) (TG), and the UCL MSci in Chemistry (HYA). This work was undertaken at UCLH/UCL, which is funded in part by the Department of Health's NIHR Biomedical Research Centres funding scheme.

\section{References}

1 H. Kwart and E. R. Evans, J. Org. Chem., 1966, 31, 410-413.

2 M. S. Newman and H. A. Karnes, J. Org. Chem., 1966, 31, 3980-3984.

3 D. W. Porter, M. Bradley, Z. Brown, R. Canova, S. Charlton, B. Cox, P. Hunt, D. Kolarik, S. Lewis, D. O'Connor, J. Reilly, C. Spanka, L. Tedaldi, S. J. Watson, R. Wermuth and N. J. Press, Bioorg. Med. Chem. Lett., 2014, 24, 72-76.

4 A. Sørensen, B. Rasmussen, S. Agarwal, M. Schau-Magnussen, T. I. Sølling and M. Pittelkow, Angew. Chem. Int. Ed., 2013, 52, 12346-12349.

5 S. Otto, Science, 2002, 297, 590-593.

6 A. Błaszczyk, M. Chadim, C. von Hänisch and M. Mayor, Eur. J. Org. Chem., 2006, 3809-3825.

7 G. Lloyd-Jones, J. Moseley and J. Renny, Synthesis, 2008, 661-689.

8 A. J. Perkowski, C. L. Cruz and D. A. Nicewicz, J. Am. Chem. Soc., 2015, 137, 15684-15687.

9 T. Broese, A. F. Roesel, A. Prudlik and R. Francke, Org. Lett., 2018, 20, 7483-7487.

10 S. K. Pedersen, A. Ulfkjær, M. N. Newman, S. Yogarasa, A. U. Petersen, T. I. Sølling and M. Pittelkow, J. Org. Chem., 2018, 83, 12000-12006.

11 T. Gendron, K. Sander, K. Cybulska, L. Benhamou, P. K. B. Sin, A. Khan, M. Wood, M. J. Porter and E. Årstad, J. Am. Chem. Soc., 2018, 140, 11125-11132.

12 J. N. Harvey, J. Jover, G. C. Lloyd-Jones, J. D. Moseley, P. Murray and J. S. Renny, Angew. Chem. Int. Ed., $2009,48,7612-7615$.

13 J. Morley. Anderson and J. K. Kochi, J. Am. Chem. Soc., 1970, 92, 1651-1659.

14 D. A. House, Chem. Rev., 1962, 62, 185-203.

15 S. Wacławek, H. V. Lutze, K. Grübel, V. V. T. Padil, M. Černík and Dionysios. D. Dionysiou, Chem. Eng. J., 2017, 330, 44-62.

16 C. L. Cruz and D. A. Nicewicz, ACS Catal., 2019, 9, 3926-3935.

17 M. J. Bollong, H. Yun, L. Sherwood, A. K. Woods, L. L. Lairson and P. G. Schultz, ACS Chem. Biol., 2015, 10, 2193-2198. 